\title{
An Unusual Bryan and Morrey Type IV Capitellum Fracture with Posterolateral Rotational Instability: A Case Report
}

\author{
Saisunder Shashank Chaganty ${ }^{1}$, G Rex Meril ${ }^{2}$, Avinash Sundass ${ }^{3}$, Deeptiman James ${ }^{4}$ \\ Learning Point of the Article: \\ Unique fracture pattern of the Capitellum characterized by loss of posterior column integrity and postero-lateral osseo-ligamentous avulsion.
}

\section{Abstract}

Introduction: Better understanding of disruption of bony and ligamentous constraints of the radiocapitellar joint in complex capitellum fracture is essential for optimizing the surgical care and outcome. This report highlights the need for modification in surgical fixation technique to address an unusual variant of this rare injury.

Case Report: We report a three-part capitellum fracture pattern with a Y-shaped split of distal humerus with a sheared anterior fragment and a single, large, non-articular posterolateral fragment in a young male patient. Articular fragment was fixed with two countersunk anterior-distal to posterior-proximal oblique screws. Additional screw was used to fix the posterolateral fragment to prevent posterolateral rotational instability. Mayo elbow performance score was 95 with no rotational instability at 2 years follow-up.

Conclusion: Additional fixation of posterolateral fragment in this three-part variant of Bryan and Morrey Type IV capitellum fracture ensured rotational stability and good function.

Keywords: Complex capitellum fracture, anterior-posterior fixation, elbow posterolateral rotatory instability, posterolateral capitellum fracture.

\section{Introduction}

Complex Type IV Bryan and Morrey capitellum fractures are uncommon elbow injuries and need prompt surgical fixation for optimal functional outcome [1]. Transmission of axial forces from the radial head shearing the capitellum and direct lateral blow from spontaneous reduction of posterolaterally subluxed radial head following fall on an outstretched hand are suggested mechanisms of injury [2]. Isolated capitellum fractures are uncommon and tend to be associated with lateral epicondyle avulsion, lateral collateral ligament (LCL) avulsion, or radial head fractures [3]. Anatomical reduction and rigid fixation with at least two lag screws with preservation of posterior soft-tissue sleeve is advocated for such fractures [4]. Coexisting injuries may need additional fixation to ensure elbow stability and are associated with poorer outcome [5].
We report a 2-year follow-up of an unusual variant of Bryan and Morrey Type IV capitellum fracture with three-part fracture pattern with a Y-shaped split of distal humerus, sheared anterior fragment, and a single, large posterolateral fragment with avulsed LCL. At 2-year follow-up, the patient had no pain, nearnormal elbow movement, and no rotational stability. Written informed consent was obtained from the patient for reporting this case.

\section{Case Report}

A 26-year-old male, manual laborer presented with pain and swelling over left elbow following a fall on an outstretched hand while driving a two-wheeler. There were diffuse swelling and tenderness over lateral elbow joint line. Forearm rotation was

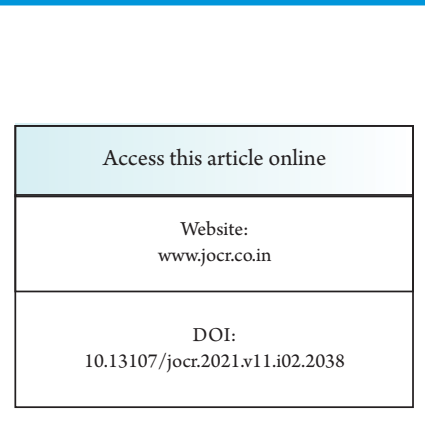

Author's Photo Gallery

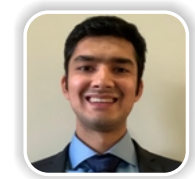

Mr. Saisunder Shashank Chaganty

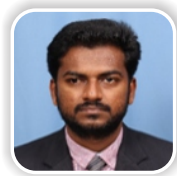

Dr. G Rex Meril

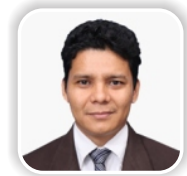

Mr. Avinash Sundass

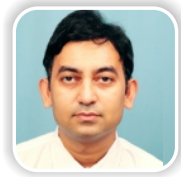

Dr. Deeptiman James
Department of Psychiatry, University of Oxford, Oxford, United Kingdom,

${ }^{2}$ Department of Orthopaedics, Christian Medical College, Vellore, Tamil Nadu, India,

${ }^{3} \mathrm{Head}$ of the department of Nursing Services, School of Nursing, Christian Hospital Mungeli, Chhattisgarh, India,

${ }^{4}$ Department of Pediatric Orthopedic, Christian Medical College, Vellore, Tamil Nadu, India.

Address of Correspondence:

Dr. Deeptiman James,

Department of Pediatric Orthopedic, Christian Medical College, Vellore, Tamil Nadu, India.

E-mail: deeptiman99james@gmail.com

Journal of Orthopaedic Case Reports | pISSN 2250-0685 | eISSN 2321-3817 | Available on www.jocr.co.in | doi:10.13107/jocr.2021.v11.i02.2038 This is an Open Access article distributed under the terms of the Creative Commons Attribution Non-Commercial License (http://creativecommons.org/licenses/by-nc/3.0) which permits unrestricted non-commercial use, distribution, and reproduction in any medium, provided the original work is properly cited. 


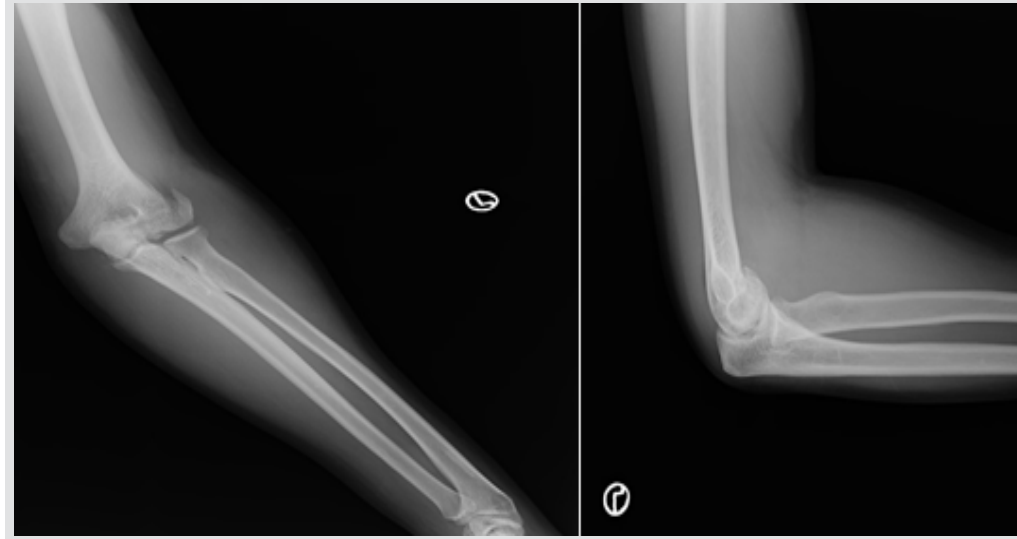

Figure 1: (a) Anteroposterior plain radiograph of the left elbow view depicting displaced Bryan and Morrey Type IV capitellum fracture. (b) Lateral view plain radiograph of the left elbow depicting capitellum fracture with double arcsign.

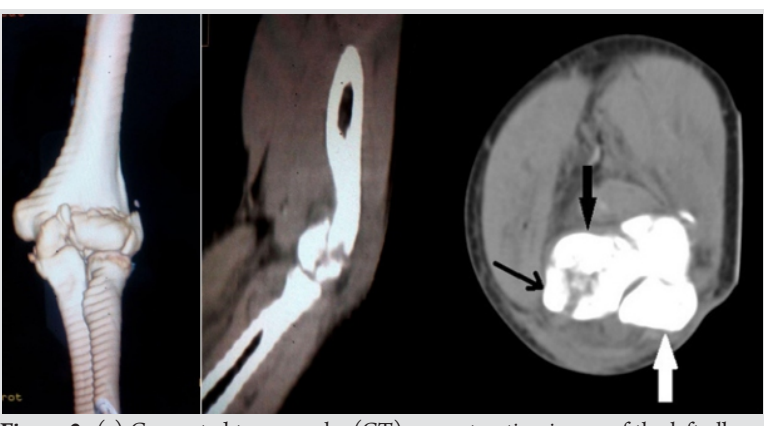

Figure 2: (a) Computed tomography (CT) reconstruction image of the left elbow demonstrates capitellum fracture extending up to the lateral trochlear ridge and avulsed fragment along lateral supracondylar ridge (b) Sagittal CT image demonstrates a Y-shaped fracture line with anterior sheared fragment and single, large posterolateral fragment. (c) Axial CT image demonstrates three-part fracture with anterior articular capitellum fragment (thick black arrow), single large posterolateral fragment including the lateral epicondyle (narrow black arrow). The white arrow points to the olecranon. associated with pain. Elbow was held in $30^{\circ}$ flexion with painful restriction of further flexion. There was no distal neurovascular deficit.

An intra-articular displaced capitellum fracture was diagnosed on plain radiographs of the elbow (Fig. 1a, b). Computed tomography (CT) images revealed a three-part capitellum fracture pattern with a Y-shaped split of distal humerus, a sheared anterior crescent-shaped fragment, and a single, large posterolateral fragment including the lateral epicondyle. The anterior crescent-shaped capitellum fragment extending up to the lateral part of trochlea was displaced proximally and laterally. The posterior arm of the Y-shaped fracture line exited the posterolateral cortex of distal humerus (Fig. 2a, b, c).

Open reduction and internal fixation (ORIF) was done through an anterolateral Kocher's interval. Posterior soft-tissue sleeve was left undisturbed. Anatomical articular reduction was temporarily held with two $1.0 \mathrm{~mm}$ Kirschner wires (Fig. 3).
Headless cannulated screws were not available at this trauma center which is a low-resource health-care setup. Instead, two divergent, countersunk, solid $3.5 \mathrm{~mm}$ cortical screws were used to stabilize the articular fragment. Both screws were applied obliquely from anterior-distal to posterior-proximal to ensure adequate far cortex purchase above the unstable posterolateral fragment and to prevent posterior column comminution (Fig. 4). An additional oblique, extra-articular screw was used to fix the posterolateral fragment. Primary LCL repair was done and additional absorbable sutures used to tag the LCL to the intact posterolateral soft-tissue sleeve. Elbow was taken through a full range of movement and valgus and rotational stability was assessed, wound was irrigated and closed over suction drain. Active-assisted elbow range of movement exercises initiated from the 7 th post-operative day.

At 3 months follow-up, fracture united and the patient regained active elbow flexion from $5^{\circ}$ to $130^{\circ}$. He complained of minor

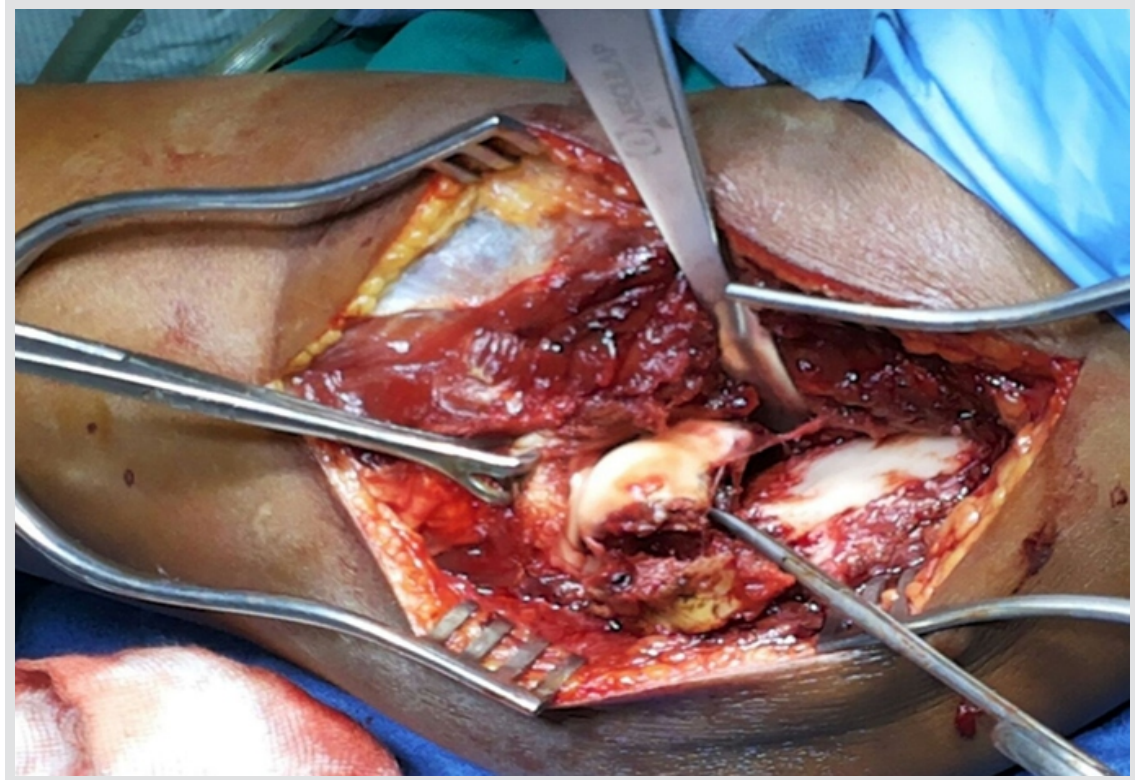

Figure 3: Intraoperative image demonstrates the anterior capitellum articular fragment and single large posterolateral fragment and avulsed lateral collateral ligament.

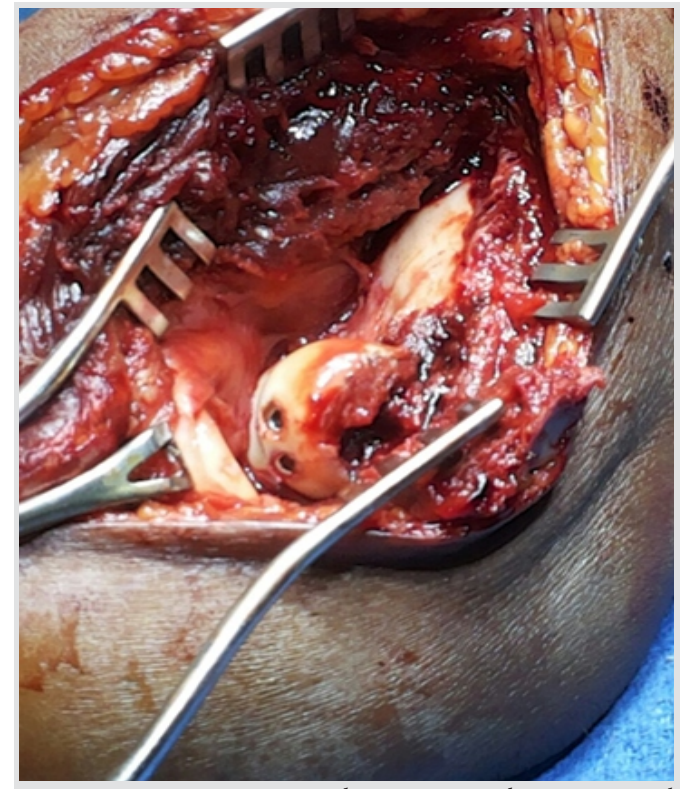

Figure 4: Intraoperative image with two countersunk $3.5 \mathrm{~mm}$ cortical screws stabilizing the articular reduction. 


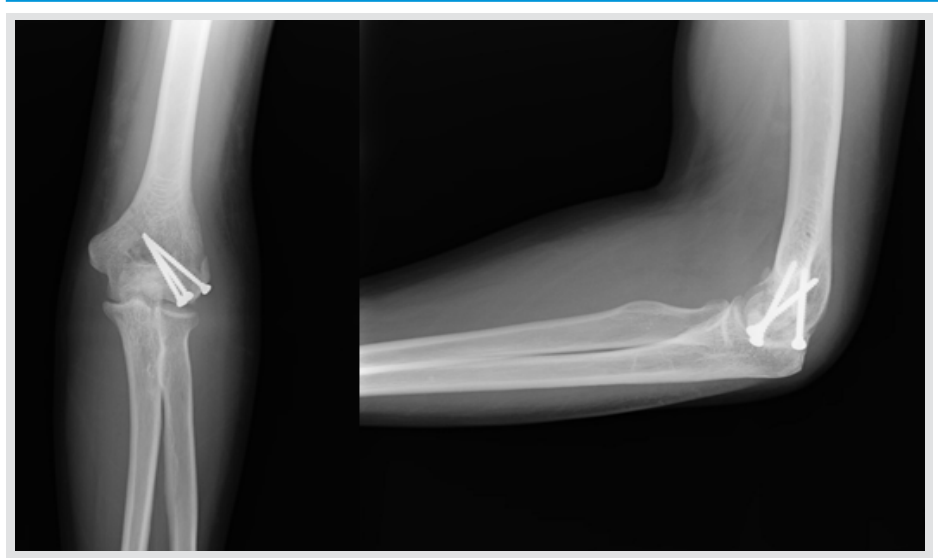

Figure 5: (a) Anterior-posterior radiograph of the left elbow at 2 years follow-up with lateral supracondylar ridge thickening but no arthritic changes. (b) Lateral plain radiograph of the left elbow at 2 years follow-up with posterior screw prominence over olecranon fossa.

pain at terminal elbow extension. Persistent restriction of $5^{\circ}$ terminal elbow extension was attributed to suspected impingement of olecranon tip against screw tip. Implant was not removed as the patient has good elbow function and was able to return to work 3 months after surgery.

At 1 year and 2 years' follow-up, elbow range of $5-130^{\circ}$ was recorded and Mayo elbow performance score was 95 . There was no valgus or posterolateral rotational instability. The patient reported no pain, full elbow flexion, minimal restriction of elbow movement at terminal extension, and no elbow instability. Minimal asymptomatic anterior heterotopic ossification (HO) was noted but it did affect elbow function (Fig. 5a, b)

\section{Discussion}

Stable fixation and anatomical articular reduction of Bryan and Morrey Type IV fracture allows early mobilization and good outcome [5]. Management of this complex fracture pattern can be associated with $\mathrm{HO}$, avascular necrosis, and chronic posterolateral rotational instability secondary to ligamentous injury or Osborne-Cotterill osteochondral lesion $[5,6]$. Posterior column instability and associated injuries have a direct bearing on the surgical outcome. However, the anatomical Bryan and Morrey classification does not address the above factors. Hence, Dubberley et al. subclassified capitellum fractures with and without posterior column comminution and Ring et al. identified associated injuries [7, 8]. Surgical approach and preferred fixation method can be determined using both these classification systems.

Our patient presented with a three-part capitellum fracture pattern with a Y-shaped split of distal humerus. The sheared anterior fragment was similar to Dubberly Type 2A. The single, large, posterior-lateral fragment included the avulsed lateral epicondyle and avulsed LCL extended to the posterior-lateral 3rd facet described by Ring et al. but just sort of the posterior column. This injury predisposed the posterior column and elbow to iatrogenic fracture and posterolateral rotational instability, respectively. This fracture pattern can neither be classified as true Dubberly Type 2A nor as Ring Type 2 or 3 because the large posterior-lateral fragment included both the second and third articular facets $[7,8]$. In view of routine clinical use of Bryan and Morrey classification, we posit to describe this fracture pattern as Bryan and Morrey Type IV (B) variant to emphasize use of CT scan for accurate diagnosis of such fracture pattern and suggest surgical fixation of both fragments with oblique screws to enable adequate posterior cortex purchase and avoid iatrogenic posterior column comminution and posterolateral rotational instability.

ORIF with Herbert (headless) screws is the recommended implant for fixation. Instrumentation for these specialized screws tends to be expensive and is not always available in lowresource health-care settings in low-middle-income countries (LMICs). Dubberley et al. recommended posteromedial screws for Type 2A fractures and additional screws or sutures to fix lateral epicondyle fractures [7]. Ruchelsman et al. and Ravishankar et al. reported good outcome with anterior to posterior screws for Bryan and Morrey Type IV fracture fixation and recommended additional mini-fragment screws for associated lateral epicondyle fractures $[4,9]$. We used three non-cannulated $3.5 \mathrm{~mm}$ countersunk cortical screws in this case and found them to be a cost effective, safe, and reliable fixation option.

Adequate imaging of complex capitellum fractures is recommended for optimum surgical planning and fixation $[3$, 10]. In this case, the $C T$ images complemented plain radiographs in detecting the Type IV (B) fracture with a single, large posterolateral fragment containing the lateral epicondyle and a small fragment avulsed from the lateral supracondylar ridge attached to the LCL. CT helped define the fracture morphology, extent of intra-articular comminution, posterior cortical integrity, and osseoligamentous injury. However, CT imaging may not be available or affordable in many LMICs trauma centers and we were fortunate to have access to CT images in this case. We suggest a high index of suspicion of a Type IV (B) fracture pattern, especially in young adults and adolescents, even when CT imaging is not feasible.

$\mathrm{HO}$, hardware prominence, osteonecrosis, arthritis, and coronal and rotational stability have been described as possible complications in Bryan and Morrey Type IV fractures $[9,10]$. Our patient did not report any pain or instability at 2 years follow-up. He complained of posterior screw impingement at extreme extension but it did not affect his function and return to work. Hastings Class I HO noted in this patient did not adversely affect his elbow function. 


\section{Conclusion}

This three-part variant of Bryan and Morrey Type IV capitellum fracture needs additional fixation to ensure rotational stability and good elbow function. A CT scan should be done for defining this fracture pattern and for planning screw positions whenever feasible. Additional care must be taken to carefully countersunk screws and determine accurate screw length and trajectory to avoid hardware-related complications.

\section{Clinical Message}

High clinical index of suspicion is essential to detect disruption of bony and ligament constraints of the radiocapitellar joint in complex capitellum fracture. Restoration of good elbow function and stability needs accurate diagnosis of fracture pattern and additional stabilization of the posterolateral fragment. Obliquely directed "anterior-distal to posterior-proximal" screws can aid in achieving stable fixation of the articular fragment with concomitant posterior column disruption.

\section{References}

1. Guitton TG, Doornberg JN, Raaymakers EL, Ring D, Kloen P. Fractures of the capitellum and trochlea. J Bone Joint Surg Am 2009;91:390-7.

2. Singh AP, Singh AP. Coronal shear fractures of distal humerus: Diagnostic and treatment protocols. World J Orthop 2015;6:867-76.

3. Rausch V, Königshausen M, Schildhauer TA, Gessmann J, Seybold D. Fractures of the capitellum humeri and their associated injuries. Obere Extrem 2018;13:33-7.

4. Ruchelsman DE, Tejwani NC, Kwon YW, Egol KA. Open reduction and internal fixation of capitellar fractures with headless screws. Surgical technique. J Bone Joint Surg Am 2009;91 Suppl2 Pt 1:38-49.

5. He SK, Xu L, Guo JH, Liao JP, Qin TW, Huang FG. The impact of associated injuries and fracture classifications on the treatment of capitellum and trochlea fractures: A systematic review and meta-analysis. Int J Surg 2018;54 Pt A:37-47.

6. Yano K, Kaneshiro Y, Sakanaka H. Acute elbow trauma similar to Osborne-Cotterill lesion, capitellar impaction fracture associated with posterolateral rotatory instability: A case report and literature review. J Orthop Sci 2019;2019:30004-1.

7. Dubberley JH, Faber KJ, Macdermid JC, Patterson SD, King GJ. Outcome after open reduction and internal fixation of capitellar and trochlear fractures. J Bone Joint Surg Am 2006;88:46-54.

8. Ring D, Jupiter JB, Gulotta L. Articular fractures of the distal part of the humerus.J Bone Joint Surg Am 2003;85:232-8.

9. Ravishankar MR, Kumar MN, Raut R. Choice of surgical approach for capitellar fractures based on pathoanatomy of fractures: Outcomes of surgical management. Eur J Orthop Surg Traumatol 2017;27:233-42.

10. Yu T, Tao H, Xu F, Hu Y, Zhang C, Zhou G. Management of isolated coronal shear fractures of the humeral capitellum with Herbert screw fixation through anterolateral approach. BMC Musculoskelet Disord 2018;19:108.

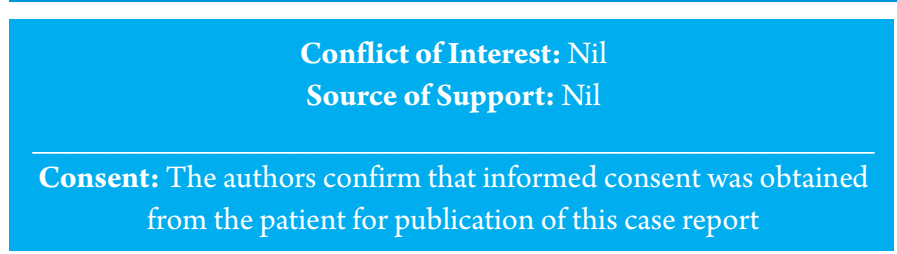

\section{How to Cite this Article}

Chaganty SS, Meril GR, Sundass A, James D. An Unusual Bryan and Morrey Type IV Capitellum Fracture with Posterolateral Rotational Instability: A Case Report. Journal of Orthopaedic Case Reports 2021 February; 11(2): 86-89. 